\title{
Análise da Relação Estatística entre as Piscinas e o Nível Socioeconômico da População de Marília - SP
}

\author{
Analysis of the Statistical Relationship between Swimming Pools and the Socioeconomic \\ Level of the Population of Marília - SP
}

Isabela do Lago Silva ${ }^{1}$, Agnes Silva de Araujo $^{2}$, Alfredo Pereira de Queiroz ${ }^{3}$

1 Universidade de São Paulo, Departamento de Geografia, São Paulo, Brasil. lagoisa@usp.br

ORCID: https://orcid.org/0000-0002-5211-6895

2 Universidade de São Paulo, Departamento de Geografia, São Paulo, Brasil. agnes.araujo@usp.br

ORCID: https://orcid.org/0000-0002-1449-8984

3 Universidade de São Paulo, Departamento de Geografia, São Paulo, Brasil. aqueiroz@usp.br

ORCID: https://orcid.org/0000-0003-4038-4953

Resumo: Dados de cobertura da terra podem ser utilizados como indicadores da diferenciação socioeconômica. Embora as piscinas residenciais sejam consideradas como equipamentos de lazer das classes sociais mais elevadas, avalia-se importante quantificar essa associação. O objetivo do artigo é relacionar a presença de piscinas ao nível socioeconômico da população de Marília - SP. A localização das piscinas foi obtida pela classificação de imagem de satélite, através de técnicas de GEOBIA, e sua associação à renda dos chefes de família foi estabelecida pela Regressão Linear Global e Regressão Geograficamente Ponderada. Os resultados confirmaram que as piscinas podem ser consideradas como um indicador da localização de chefes de famílias renda elevada. O modelo foi capaz de prever a localização da população de alta renda (acima de 10 salários mínimos) em 68\%. O modelo mostrou melhor ajuste nas residências unifamiliares horizontais (casas), mas fraco desempenho nas localidades centrais da cidade, mais verticalizada e com poucas piscinas por lote.

Palavras-chave: Equipamento de lazer. Renda. Cobertura da terra. Regressão.

\begin{abstract}
Land cover data may be used as indicators of socioeconomic differentiation. Although residential pools are considered as leisure equipment for the highest social classes, it is important to quantify this association. The aim of this paper is to relate the presence of swimming pools to the socioeconomic level of the population of Marília - SP. The geographical location of the swimming pools was obtained by the classification of satellite image, using GEOBIA techniques, and the association with the income of the heads of households was established by the Global Linear Regression and Geographical Weighted Regression. The results confirmed that the swimming pools can be an indicator of the location of high-income families. The model was able to predict the location of the high-income families (income above 10 minimum wages) at $68 \%$. The model showed a better fit in horizontal single-family houses, but poor performance in the central locations of the city, with more vertical dwellings and with few swimming pools per lot.
\end{abstract}

Keywords: Leisure equipment. Income. Land cover. Regression.

\section{INTRODUÇÃO}

Dados do uso e cobertura da terra são essenciais para o planejamento urbano, regional e monitoramento ambiental (LIU et al., 2017) e imprescindíveis para as pesquisas socioeconômicas e ambientais (STÜRCK; SCHULP; VERBURG, 2015). Atualmente, os métodos baseados em objetos são muito adotados para classificar imagens e mapear a cobertura da terra. A segmentação dos objetos (grupos de pixels) permite a 
extração de recursos discriminativos (textura, forma e característica espectral) dos fenômenos pesquisados e do contexto das adjacências (ZHANG et al., 2020).

A Análise de Imagens Baseada em Objetos Geográficos (sigla em inglês GEOBIA) permite elaborar classificações a partir da textura e do padrão espacial dos objetos (HAY; CASTILLA, 2006). Esse conjunto de relações espaciais e estatísticas melhora a qualidade da classificação final, otimizando o processo (BURNETT; BLASCHKE, 2003). Dentre as diversas aplicações de GEOBIA, destacam-se as de mapeamento de uso e cobertura da terra realizados por Costa et al. (2018), Ma et al. (2017), Mo et al. (2007), Kong, Xu e Wu (2006), e Zhang, Song e Zhou (2005).

De acordo com Jensen e Cowen (1999), indicadores como valor da casa, renda mediana da família e número médio de quartos podem ser estimados pela extração dos atributos urbanos de imagens de satélite. Avelar, Zah e Tavares (2009) usaram imagens de alta resolução e Sistemas de Informações Geográficas (SIG) para identificar zonas socioeconômicas homogêneas de Lima (Peru). Constataram que nas regiões ocupadas pela população de nível socioeconômico mais elevado os terrenos se caracterizaram pela presença de áreas verdes, corpos d'água, ruas pavimentadas e edifícios de alta qualidade. E, nas regiões mais carentes, predominou a ocorrência de solos expostos e edifícios de baixa qualidade edilícia.

Souza (2003) relacionou a densidade de edificações com o nível socioeconômico da população de São José dos Campos (SP). Souza et al. (2007) verificaram que os locais habitados pela população de alta renda possuíam ruas largas, pavimentadas e arborizadas, presença de piscinas e de grandes construções. Em contrapartida, os habitados pela população de baixa renda se caracterizaram por ruas estreitas, pavimentadas e com taxa de arborização reduzida. Para Luchiari (2001), a vegetação intraurbana e o tamanho das propriedades residenciais são indícios da presença de população de alta renda. Salim e Luchiari (2013) observaram que a densidade de telhados de amianto poderia ser considerada como indicador de presença da população de menor poder aquisitivo.

De acordo com Goudard, Oliveira e Gerente (2015) existe uma associação entre a presença de UBS (Unidades Básicas de Saúde) e a concentração da população de baixa renda em Joinville-SC. Para os autores, a renda exerceria um papel fundamental na distribuição das UBSs, uma vez que a população atendida por esse serviço é predominantemente a de menor poder aquisitivo.

Kim et al. (2011a) adotaram a GEOBIA para identificar as piscinas, potencial habitat de mosquitos (culex), que transmitem o vírus do Nilo Ocidental, na Califórnia (EUA). Com propósito análogo, McFeeters (2013) considerou fundamental usar imagens de satélite de alta resolução, integradas ao SIG, para identificar as piscinas (criadouros de mosquito). Vidal, Domene e Sauri (2011) estimaram o número, as características e o consumo de água das piscinas residenciais de Barcelona. Llausàs et al. (2019) avaliaram, por Geobia, a qualidade dos dados cadastrais sobre o número, a localização e o tamanho das piscinas de três áreas da Catalunha.

O objetivo deste artigo é relacionar a presença de piscinas e o nível socioeconômico da população de Marília. A hipótese é que existe uma associação positiva e significativa entre a presença de piscinas e a população de elevado nível socioeconômico.

Embora a correlação dessas variáveis possa ser considerada óbvia, as pesquisas espanholas salientam aspectos que minimizam essa evidência, mesmo que as diferenças entre os contextos urbanos de Marília e da Catalunha sejam elevadas. Para Llausàs et al. (2019), as famílias de renda média mais baixa tendem a ter piscinas permanentes menores e maior quantidade de piscinas temporárias. Vidal, Domene e Sauri (2011) consideraram que as piscinas não deveriam ser consideradas como elemento de refinamento residencial apenas das famílias de nível socioeconômico mais elevado, em decorrência da variedade e heterogeneidade das características das piscinas. Para os autores, "a diversificação nos tipos, tamanhos e custos abriu o mercado de piscinas para segmentos maiores da população (p. 70)". E "a relação entre renda e piscinas foi moderada no que diz respeito à densidade das piscinas, mas não foi significativa em relação ao tamanho ou número de piscinas / 1000 pessoas" (p.73).

A questão motriz desta investigação é analisar em que medida os dados pontuais das piscinas, extraídos a partir de técnicas de Sensoriamento Remoto, podem ser utilizados como proxies da localização da população de alto nível socioeconômico. Também contribui ao explorar procedimentos alternativos para reduzir a defasagem dos dados socioeconômicos nos períodos pré-censitários. 


\section{2 ÁREA DE ESTUDO}

A cidade de Marília (Figura 1) é uma das mais importantes cidades do Oeste Paulista. O município possui uma área de $1,170.174 \mathrm{~km}^{2}$, que abrigava uma população de 216,745 habitantes (IBGE, 2010), sendo que $96 \%$ desse total residia na área urbana (SEADE, 2010).

Figura 1- Localização da área de estudo.

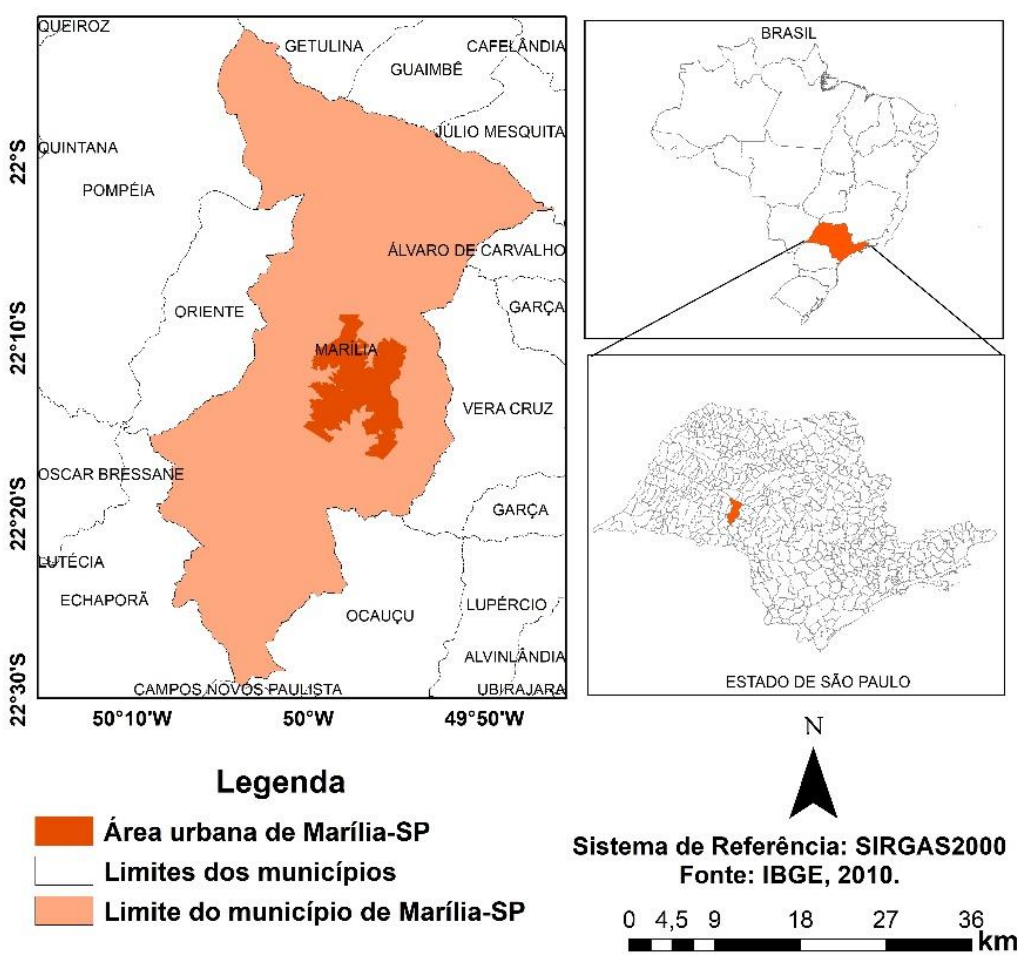

Fonte: Os autores (2021).

Marília, como outras cidades do oeste paulista, consolidou-se a partir do terminal ferroviário e desde então vem se expandindo tanto em termos espaciais quanto populacionais. Pelos diversos processos de reestruturação urbana mais recentes como o surgimento de shoppings centers (SPOSITO; GÓES, 2013), condomínios fechados (ZANDONADI, 2008), constituição de espaços de exclusão (NUNES, 2007), de desigualdade social (MELAZZO, 2006) e segregação (ARAUJO; BARROS; QUEIROZ, 2018), Marília apresenta características de cidade média (SPOSITO, 2007; SPOSITO; GÓES, 2013).

Outro aspecto relevante é a sua localização. Seus limites ocorrem entre as coordenadas geográficas $22^{\circ} 00^{\prime}$ e $22^{\circ} 30^{\prime}$ de latitude sul e $49^{\circ} 30^{\prime}$ e $50^{\circ} 00^{\prime}$ de longitude oeste, portanto, em um clima subtropical (KOTTEK et al., 2006) com temperaturas anuais médias de $26,3^{\circ} \mathrm{C}$, podendo chegar a máximas de $39^{\circ} \mathrm{C}$ no verão (CPTEC/INPE, 2020). O clima quente, especialmente no verão, parece influenciar a significativa ocorrência de piscinas na área urbana.

\section{METODOLOGIA}

Os dois principais eixos metodológicos estão associados à Análise de Imagens Baseada em Objetos Geográficos (GEOBIA), para o mapeamento das piscinas, e à correlação estatística e espacial, que associa a densidade de piscinas às variáveis de renda dos chefes de família. As principais referências bibliográficas para a GEOBIA foram Aboud Neta, Souza e dos Santos (2018), Kressler e Steinnocher, (2006) e Baatz e Schäpe (2000). A regressão linear foi baseada em Anselin (1995) e, a Regressão Geograficamente Ponderada (GWR), em Fotheringham, Charlton e Brunsdon (2001). 


\subsection{Procedimentos operacionais e técnicos}

A ilustração dos procedimentos da pesquisa pode ser observada na Figura 2. Suas especificações estão descritas nos itens seguintes.

Figura 2 - Procedimentos da pesquisa.

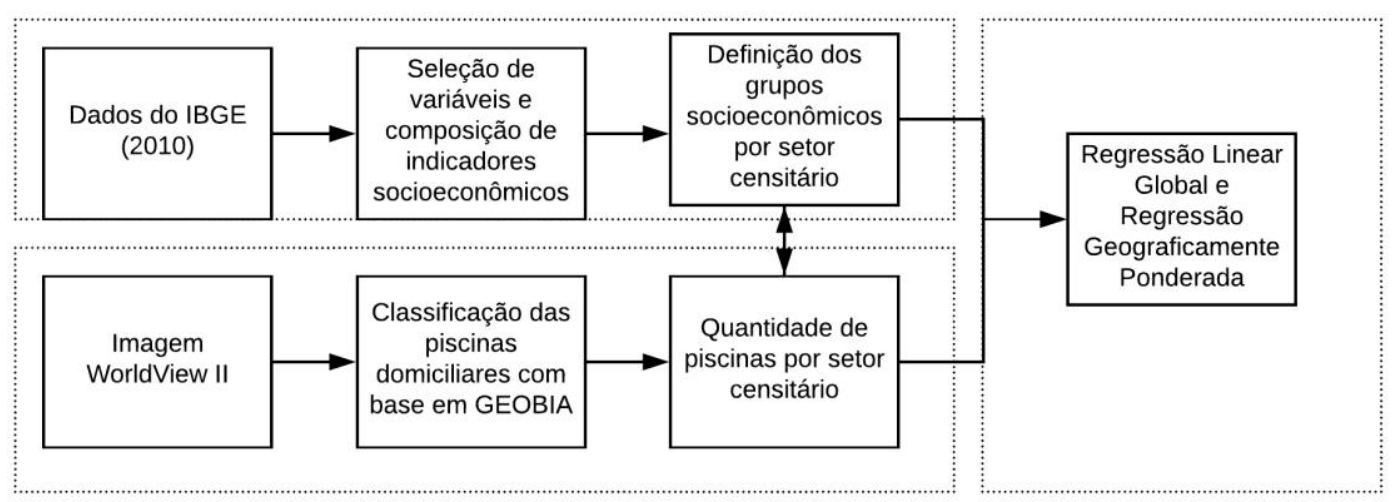

Fonte: Os autores (2021).

\subsection{Determinação dos grupos socioeconômicos}

A determinação dos grupos socioeconômicos baseou-se nas variáveis de renda dos chefes de família inclusas nos dados do Universo do Censo do IBGE de 2010 (salário mínimo na data de referência era de $\mathrm{R} \$ 510,00)$. Esses dados estão agregados por setor censitário, que é a menor unidade de agregação disponível. Foram determinados seis grupos socioeconômicos, conforme Feitosa et al. (2007). Os grupos são apresentados na Tabela 1.

Tabela 1- Grupos socioeconômicos.

\begin{tabular}{c|c}
\hline Grupo & Renda \\
\hline 1 & Sem rendimento (SR) \\
2 & De 1 a 2 salários mínimos (SM) \\
3 & De 2 a 5 salários mínimos (SM) \\
4 & De 5 a 10 salários mínimos (SM) \\
5 & De 10 a 20 salários mínimos (SM) \\
6 & Acima de 20 salários mínimos (SM) \\
\hline
\end{tabular}

Fonte: Os autores (2021).

\subsection{Classificação das piscinas}

A imagem do satélite WorldView-II, de 18/08/2010, georreferenciada e ortorretificada, foi utilizada para a classificação das piscinas de Marília. Suas características são: 2 metros de resolução espacial nas bandas multiespectrais e 50 centímetros na banda pancromática, resolução espectral com as três bandas do visível $R G B$ e do infravermelho próximo - NIR.

A imagem foi segmentada a partir de um algoritmo de segmentação multirresolução, o Fractal Evolution Approach - FNEA, processado no software eCognition da Trimble®. Os parâmetros utilizados para segmentação estão descritos na Tabela 2. 
Tabela 2- Segmentação multirresolução.

Peso atribuído às bandas da Imagem (Image layers weights)

$$
\text { Forma (Shape) }
$$

Parâmetro de Escala (Scale Parameter)

Compacidade (Compactness)

Resultado da segmentação

\section{$1,1,2,2,1$}

0,2

20

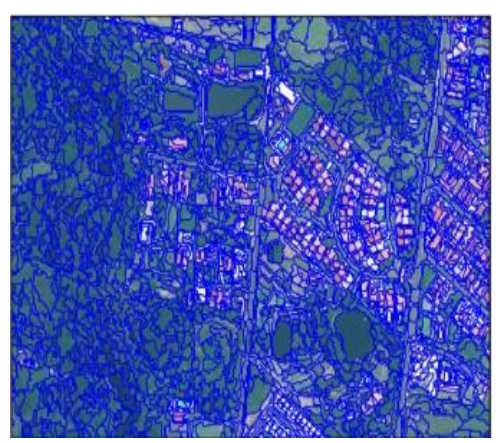

Fonte: Araujo (2015).

O parâmetro Image Layers Weights refere-se ao peso dado à cada um dos layers (bandas do azul, vermelho, verde, infravermelho próximo e pancromática). O parâmetro Shape é composto pela compacidade e pela suavidade das bordas. De acordo com Kressler e Steinnocher (2006), quanto maior o valor atribuído à geometria, menor é o valor das informações espectrais. Altos valores de geometria são usuais quando o objeto a ser classificado possui atributos espectrais conflitantes com outros. O parâmetro Compactness ressalta o aspecto geométrico do objeto. Valores elevados de compacidade contribuem para a segmentação de objetos que representam as construções antrópicas e a suavidade mais alta melhora a segmentação das feições naturais (KRESSLER; STEINNOCHER, 2006).

Os limiares e valores da classificação das piscinas estão apresentados na forma de árvore de decisão (Figura 3) e os atributos quantificáveis, com definições e formulações matemáticas, estão descritos na Tabela 3. Ressalta-se que a construção da árvore de decisão se baseou na pesquisa de Araujo e Queiroz (2018) e na análise exploratória dos atributos quantificáveis.

Figura 3 - Árvore de decisão da classificação de piscinas.

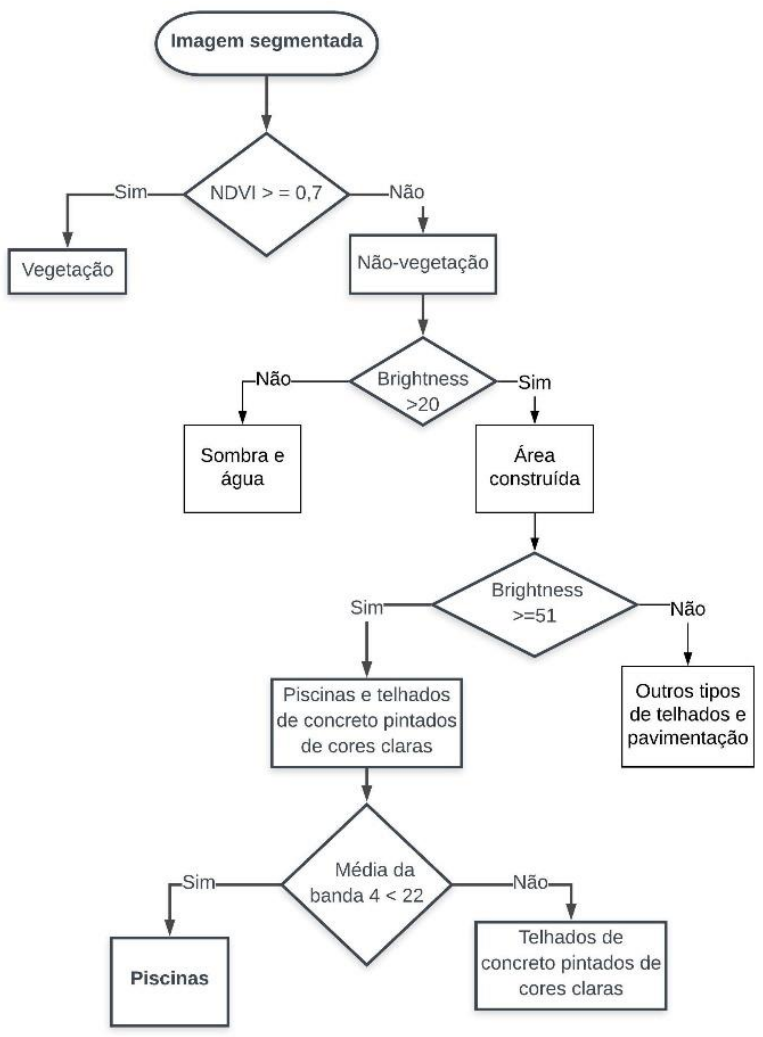

Fonte: Araujo e Queiroz (2018). 
Tabela 3- Atributos quantificáveis.

\begin{tabular}{|c|c|c|c|}
\hline Tipo de atributo & Atributo quantificável & Formulação matemática & Descrição \\
\hline \multirow{3}{*}{ Espectral } & $\begin{array}{c}\text { Índice de Vegetação por Diferença } \\
\text { Normalizada (NDVI) }\end{array}$ & $\mathrm{NDVI}=$ média $\left(\frac{N I R-R E D}{N I R+R E D}\right)$ & $\begin{array}{l}\text { O NDVI é um valor entre - } \\
\qquad 1,0 \mathrm{e}+1,0\end{array}$ \\
\hline & 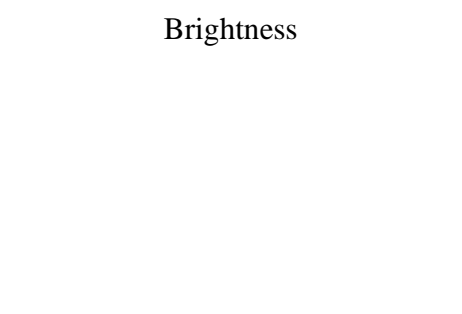 & $\mathrm{B}=\frac{1}{n_{v i s}} \sum_{i=1}^{n_{v i s}} C_{i(v i s)}^{-}$ & $\begin{array}{c}\text { B é o brilho médio de um } \\
\text { objeto e } C_{i}^{-} \text {(vis) é a soma de } \\
\text { todos os valores médios de } \\
\text { brilho nas bandas visíveis } \\
\text { dividido pelo número } \\
\text { correspondente de bandas } \\
\text { visíveis } n_{v i s}\end{array}$ \\
\hline & Média da banda 4 (NIR) & Média da banda 4= média (NIR) & $\begin{array}{c}\text { A média da banda } 4 \text { é o } \\
\text { valor médio da resposta } \\
\text { espectral de um objeto na } \\
\text { banda } 4 \text { (NIR) }\end{array}$ \\
\hline
\end{tabular}

Fonte: Os autores (2021).

Após classificados, os polígonos das piscinas foram transformados em informações pontuais, através das quais foi computada a densidade kernel.

\subsection{Regressão}

De acordo com Weisberg (2005), o modelo de regressão linear simples analisa a relação entre duas variáveis. Determina a relação entre as variáveis dependente (y) e a independente ou explicativa (x). Para a associação entre as variáveis dependente e independente, uma regressão linear global (NETTER; WASSERMAN, 1977) foi elaborada. A Eq. (1) que descreve a relação estatística entre as variáveis foi:

$$
R E N D A=\beta \theta+\beta 1 . P I S C I N A S+\varepsilon
$$

onde:

RENDA representa a quantidade de chefes de família com uma determinada renda (variável dependente); $\beta$ são os parâmetros de interceptação e inclinação do modelo de regressão; PISCINAS representa a quantidade de piscinas (variável independente); $\varepsilon$ é a soma dos resíduos no modelo.

\subsection{Regressão geograficamente ponderada}

Proposta por Brunsdon, Fotheringham e Charlton (1996), a Regressão Geograficamente Ponderada (GWR) foi desenvolvida para refinar a descrição estatística dos fenômenos geográficos. É considerada uma estatística espacial local, pois é utilizada para ajustar um modelo de regressão para cada ponto de um conjunto de dados, ponderando as observações em função da distância de um ponto centroidal (CARVALHO et al., 2006). Sousa et al. (2012) afirmam que o GWR pode ser considerada como um aperfeiçoamento da regressão linear global, pois incorpora o papel do espaço em suas análises.

Esse método de regressão espacial parte do pressuposto de que o espaço é heterogêneo ou nãoestacionário e a relação entre as variáveis dependentes e independentes assumem padrões mais ou menos regionalizados, portanto, realiza uma regressão local para cada unidade de análise levando em conta os vizinhos com base num kernel fixo ou adaptativo (FREITAS; SANTOS, 2011).

No modelo, assume-se que as informações mais próximas do ponto de regressão têm maior probabilidade de influenciá-lo. Essa ponderação é feita pela função kernel espacial, cuja integral soma 1, semelhante a uma função de densidade de probabilidade (ANSELIN, 2001). O kernel usa distância entre dois pontos geográficos representando duas regiões e um parâmetro da largura da banda para determinar um peso 
entre essas duas regiões, que é inversamente relacionado à distância geográfica (ANSELIN, 2001). A Eq. (2) descreve a relação estatística e espacial entre as variáveis:

$$
R E N D A i=\beta \theta(u i, v i)+\beta 1(u i, v i) \cdot P I S C I N A S i+\varepsilon i
$$

onde:

RENDAi representa a quantidade de chefes de família com uma determinada renda no setor $i$ (variável dependente); $\beta$ são os parâmetros de intercepção e inclinação do modelo de regressão a serem estimados respectivamente nas localizações $\theta$ e 1; PISCINASi representa a quantidade de piscinas (variável independente) no setor censitário $i$; $\varepsilon i$ é a soma dos resíduos do modelo de regressão local em $i$.

Determinou-se, através do algoritmo Golden Section Search (FOTHERINGHAM; CHARLTON; BRUNSDON, 2001) que a largura de banda de 45 vizinhos mais próximos estabelece resultados otimizados para o GWR utilizando um kernel adaptativo.

No entanto, testes prévios de pseudossignificância são fundamentais, pois a GWR pode sugerir uma associação estatística indevida (FOTHERINGHAM; CHARLTON; BRUNSDON, 2001; XUAN; LI, 2012). Se os testes indicarem autocorrelação espacial significativa entre as variáveis, os procedimentos são: 1) aplicar os resíduos no modelo de regressão (GWR) e criar um Spatial Lag Model (SAR), onde a autocorrelação é atribuída à variável resposta, 2) atribuir a autocorrelação ao erro do modelo (Spatial Error Models) (ANSELIN, 2001). Em ambos os casos, assume-se que considerar os efeitos da autocorrelação contribui para aproximar o modelo da realidade (FOTHERINGHAM; CHARLTON; BRUNSDON, 2001; XUAN; LI, 2012).

\subsection{Testes de dependência espacial}

A autocorrelação espacial avalia se o espaço exerce influência nos valores da variável analisada, ou seja, se observações espacialmente próximas apresentam valores parecidos (ANSELIN, 1995). Os testes estatísticos de diagnóstico utilizados foram: Índice de Moran, Multiplicadores de Lagrange Robusto (Spatial Lag e Error) e Jarque-Bera.

O Índice de Moran representa a autocorrelação espacial ou o nível de interdependência espacial entre os dados analisados (ANSELIN, 1995; MONTEIRO, 2004). Se confirmada a autocorrelação entre qualquer uma das variáveis e pseudossignificância, torna-se necessário escolher um modelo espacial que melhor descreva a relação de dependência entre as variáveis (ANSELIN, 1988). Ainda segundo o autor, o Multiplicador de Lagrange Robusto (LM) é um teste consistente, que compara de forma hipotética os modelos com a regressão convencional por porcentagem de confiança, executando regressão dos resíduos em relação às variáveis originais e em relação aos resíduos das áreas vizinhas (ANSELIN, 1988).

O teste de Jarque-Bera foi utilizado para determinar se os dados têm assimetria a uma distribuição normal (JARQUE; BERA, 1980). Quanto maior for sua distância de zero, mais significativa será a anormalidade da distribuição dos dados no espaço (JARQUE; BERA, 1980).

\section{RESULTADOS E DISCUSSÃO}

\subsection{Classificação das piscinas}

O resultado da classificação das piscinas está apresentado na Figura 4. Foi possível extrair 806 piscinas, das quais 12 foram eliminadas por pertenceram aos clubes ou às instituições do Sistema S (SESI, SENAI, etc.). 
Figura 4- Resultado da classificação de piscinas domiciliares.

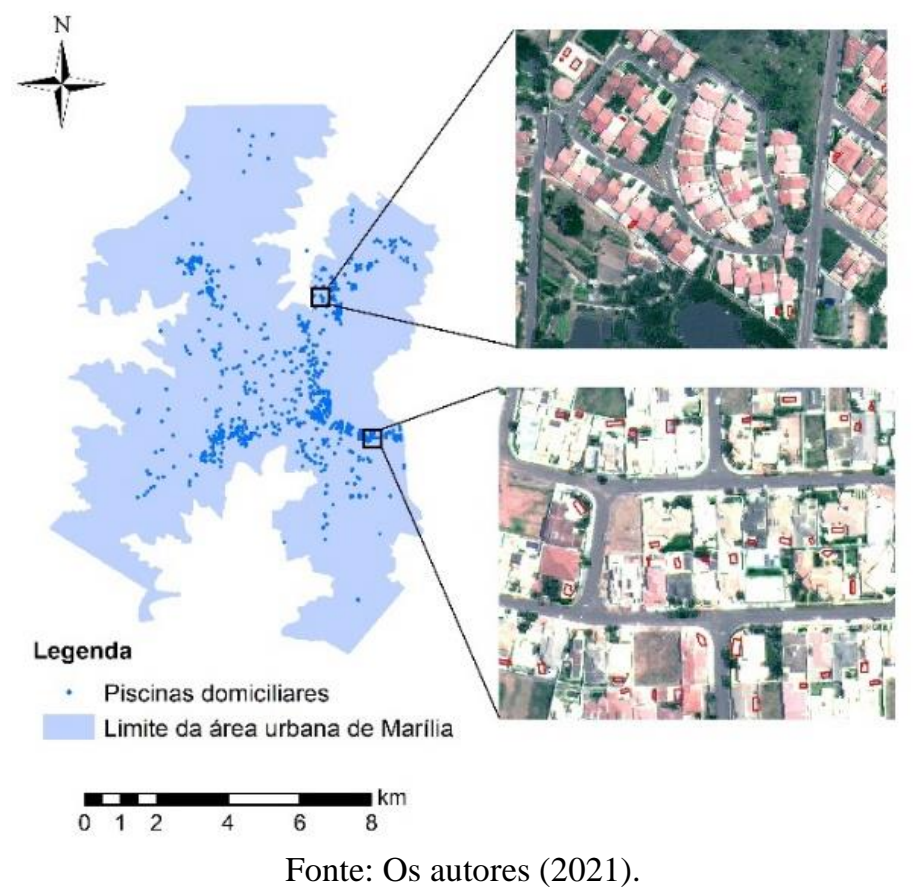

Para avaliação e validação dos resultados, 110 pontos de verdade de campo foram utilizados para cálculo do coeficiente kappa, recomendado para avaliação de classificação de imagens de Sensoriamento Remoto (CONGALTON, 2009). O percentual de acerto foi de $91 \%$ resultando em um kappa de 0,83, considerado de excelente qualidade de acordo com a proposta de Landis e Koch (1977).

Apesar do alto valor de kappa, o índice penalizou em $50 \%$ as probabilidades de concordância aleatória. Limitações do coeficiente kappa na avaliação de produtos cartográficos foram extensivamente discutidas pela literatura (PONTIUS JR; MILLONES, 2011; KEER; FISCHER; REULKE, 2015; FOODY, 2020) e diversas foram as sugestões para substituição do índice (MA; REDMOND, 1995; DOU et al., 2007; HAGENZANKER, 2009; LI; GOU, 2013). No entanto, ainda não existem propostas amplamente aceitas pela literatura. O índice ainda é utilizado para acessar acurácia de mapeamentos temáticos por GEOBIA (KIM et al., 2011a; FRANCISCO; ALMEIDA, 2012; BELGIU; DRĂGUȚ; STROBL, 2014; COLARES et al., 2015; MAXWELL et al., 2019).

\subsection{Regressão linear global}

Os resultados da regressão linear global estão expressos na Tabela 4.

Tabela 4- Regressão Linear Global.

\begin{tabular}{c|c|c|c|c|c|c}
\hline Rendimento & Sem renda & 1 a 2 SM & 2 a 5 SM & 5 a 10 SM & 10 a 20 SM & $>$ de 20 SM \\
$\mathbf{R}^{2}$ & 0,00047143 & 0,1061653 & 0,0292727 & 0,3792827 & 0,5267062 & 0,588968096 \\
R ajustado & $-0,0030234$ & 0,10304 & 0,0258786 & 0,3771123 & 0,5250513 & 0,587530922 \\
Desvio padrão & 18,6271798 & 57,789781 & 35,719427 & 18,913134 & 8,9088543 & 3,287665556 \\
\hline
\end{tabular}

Fonte: Os autores (2021).

Essa tabela mostrou $\mathrm{R}^{2}$ superior a 0,5 , para as duas faixas rendas acima de $10 \mathrm{SM}$. O modelo apresentou uma relação positiva e estatisticamente significante ( $p$-value $=0,0000$ ) entre quantidade de piscinas e de chefes de famílias com alta renda. Os valores de $\mathrm{R}^{2}$ indicam que o modelo é capaz de descrever 52 e $58 \%$ da localização dos chefes de família com renda entre 10 e 20 SM e acima de 20 SM, respectivamente. Nessas duas faixas de renda, o desvio padrão mostrou que o modelo pode errar $0,89 \%$ e $0,32 \%$, para mais ou para menos, com mais de $95 \%$ de confiança.

Para avaliar a existência de dependência espacial entre as variáveis, foram realizados testes diagnósticos (Tabela 5). 
Tabela 5- Diagnóstico para dependência espacial.

\begin{tabular}{c|c|c|c|c}
\hline \multirow{2}{*}{} & \multicolumn{3}{|c}{ Rendimento } \\
\cline { 2 - 5 } & \multicolumn{2}{|c}{ De 10 a 20 SM } & \multicolumn{2}{c}{ Acima de 20 SM } \\
\cline { 2 - 5 } Índice de Moran & & p-value & p-value \\
LM Robusto (LAG) & 82,460 & 0 & 56,416 & 0 \\
LM Robusto (ERROR) & 376,677 & 0 & 340,708 & 0 \\
Jarque-Bera & 0,7427 & 0,58881 & 0,0253 & 0,87359 \\
\hline
\end{tabular}

Fonte: Os autores (2021).

A dependência espacial entre as duas faixas de renda apontou valores positivos $(82,46$ e 56,41) e significativos ( $\mathrm{p}$-value $=0,0000$ ) para o Índice de Moran. O I de Moran indicou ausência de hipótese nula de autocorrelação espacial entre os dados, que significa que há autocorrelação espacial. Os testes de LM Robusto $(L A G)$ apontaram uma significativa autocorrelação da variável dependente para as duas faixas de renda analisadas ( $\mathrm{p}$-value $=0,0000$ ), apontando para a necessidade desenvolver um Spatial Lag Model, atribuindo este comportamento à variável dependente.

O teste de Jarque-Bera mostra se os erros da regressão linear possuem distribuição normal no espaço, ou seja, se têm comportamento semelhante em toda a área, baseado em diferenças entre assimetria e curtose na distribuição das variáveis em relação à normal. Quando os valores indicam um alto e significativo (p-value= 0) grau de autocorrelação nos erros, a hipótese nula é rejeitada (FERREIRA, 2006).

\subsection{Densidade de piscinas e renda acima de $10 \mathrm{SM}$}

Considerando os resultados da regressão linear global e os testes de diagnóstico da dependência espacial, constatou-se a existência de relação estatística entre a presença de piscinas e as rendas mais elevadas. A Figura 5 apresenta três mapas que demonstram como os dados da densidade de piscinas e do número de responsáveis com renda acima de $20 \mathrm{SM}$ e entre 10 e $20 \mathrm{SM}$ se encontram agrupados e concentrados em determinadas localidades na área de estudo.

As localidades que possuem maior concentração de piscinas, são aquelas que abrigam o maior número de chefes de família com renda acima de 10 SM (Figura 5). Nota-se que estão concentrados no eixo nordestesudoeste da cidade. De acordo com Araujo, Barros e Queiroz (2018), a população com nível socioeconômico mais elevado localiza-se no setor centro-leste, que apresenta concentração de condomínios fechados de casas, de alto e médio padrão, e de chácaras, com alta densidade de piscinas. A população de nível socioeconômico mais baixo, em contrapartida, está segregada na região sul, onde há assentamentos urbanos irregulares e conjuntos habitacionais (ARAUJO; BARROS; QUEIROZ, 2018), com ausência de piscinas (ARAUJO; QUEIROZ, 2018). 
Figura 5 - Relação entre a densidade de piscinas domiciliares e renda acima de 10 SM.

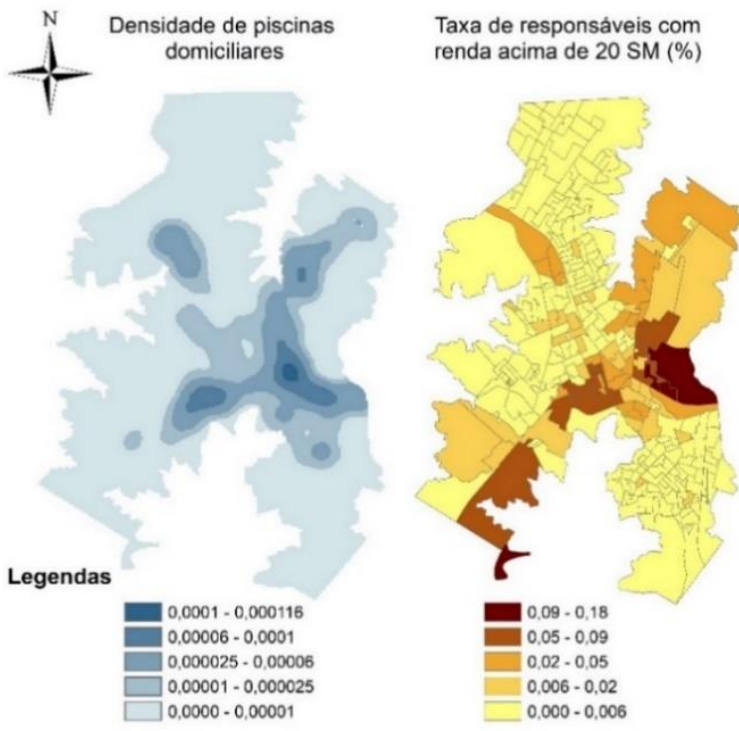

Fonte: Os autores (2021).

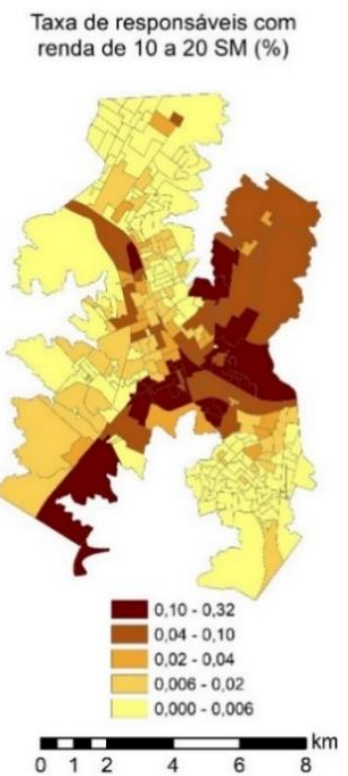

\subsection{GWR}

A regressão geograficamente ponderada foi usada para estimar se a relação entre as variáveis se altera de forma heterogênea. Os resultados são apresentados na Tabela 6 e nas Figuras 6 e 7.

Tabela 6- Regressão Geograficamente Ponderada.

\begin{tabular}{c|c|c}
\hline \multirow{2}{*}{$\mathrm{R}^{2}$} & \multicolumn{2}{c}{ Rendimento } \\
\cline { 2 - 3 } & De 10 a 20 SM & Acima de 20 SM \\
R ajustado & 0,748805 & 0,792711 \\
& 0,707998 & 0,759037 \\
\hline
\end{tabular}

Fonte: Os autores (2021).

Figura 6 - Resultados do modelo GWR acima de 20 SM.

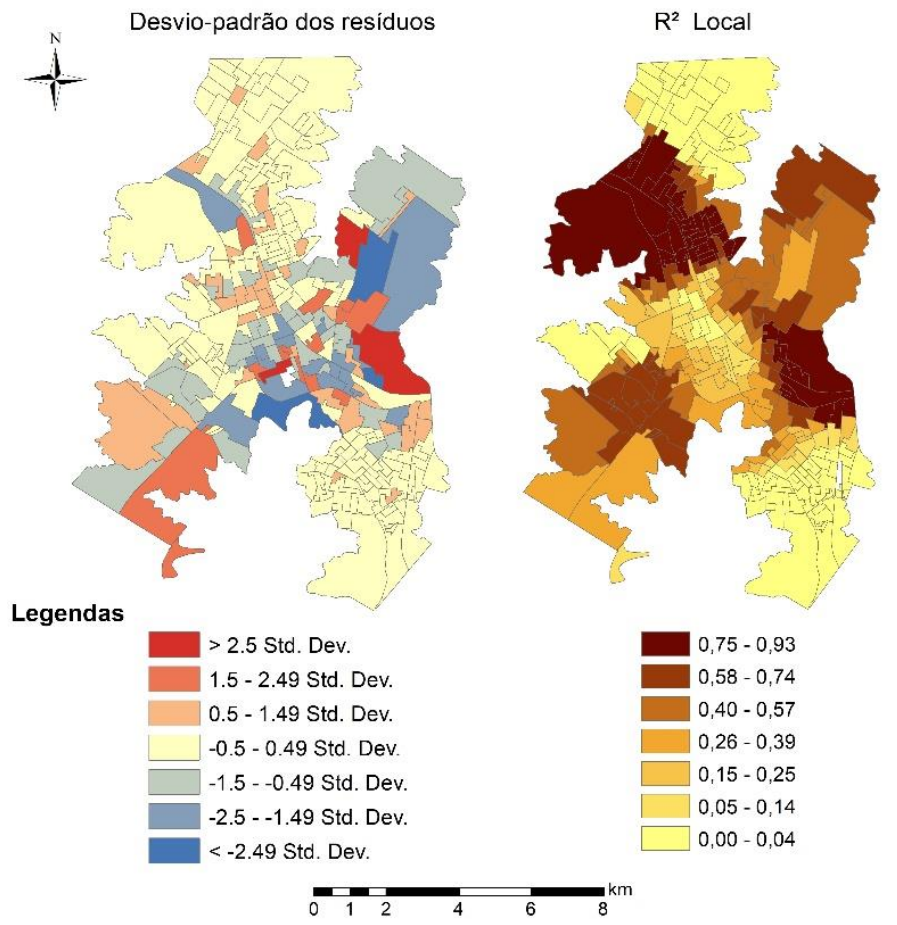

Fonte: Os autores (2021). 
Figura 7 - Resultados do modelo GWR entre 10 e 20 SM.

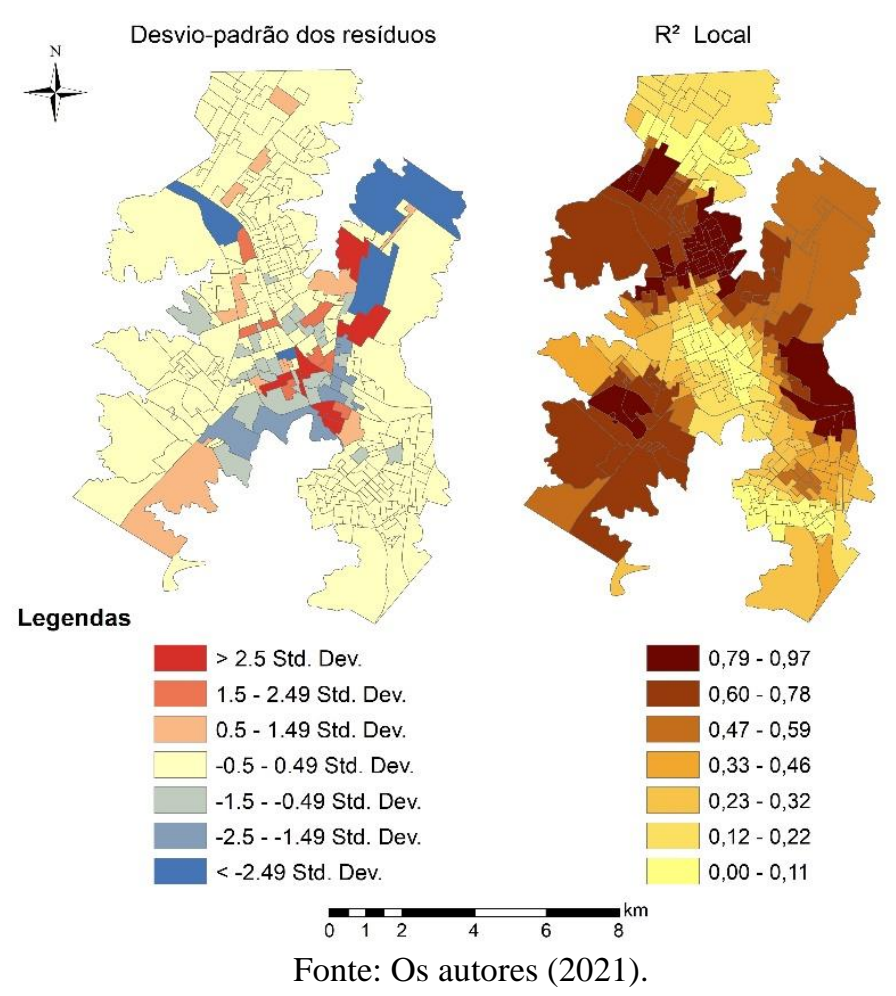

Os valores do $\mathrm{R}^{2}$ foram de 0,748805 e 0,792711 para as faixas de 10 a $20 \mathrm{SM}$, e acima de $20 \mathrm{SM}$ respectivamente. Isso indica que o modelo foi capaz de prever a renda pela presença de piscinas em até $74 \%$ para a faixa de 10 a $20 \mathrm{SM}$ e $79 \%$ para as faixas acima de $20 \mathrm{SM}$, se considerarmos a dependência espacial entre as variáveis. Em suma, a distribuição espacial é crucial para um melhor ajuste no modelo.

As Figuras 6 e 7 mostraram os resultados do desvio-padrão dos resíduos e o coeficiente local de determinação ( $\mathrm{R}^{2}$ local). O desvio-padrão indicou as regiões nas quais o modelo superestimou (em vermelho) ou subestimou (em azul) a predição da localização dos chefes de família com alta renda com base na localização das piscinas. Nota-se que as regiões do eixo noroeste-sudoeste possuem mais erros estimados, que coincide com as áreas de maior diversidade social (ARAUJO; BARROS; QUEIROZ, 2018).

Os resultados espaciais do $\mathrm{R}^{2}$ local indicam as áreas onde o modelo conseguiu prever uma porcentagem maior dos chefes de família com alta renda (tons escuros). Em ambos os casos, estas localidades concentram as residências do tipo casa ou chácaras nas quais existe, em média, uma piscina por domicílio ou condomínios de casas em que há mais de uma piscina nas áreas de lazer (ARAUJO; QUEIROZ; 2018). São áreas onde a população de alta renda está concentrada na área de estudo (ARAUJO; BARROS; QUEIROZ, 2018).

Por outro lado, o modelo obteve um fraco desempenho na região central da cidade e nas localidades dos extremos sul e norte. Acredita-se que o modelo tenha subestimado a população de alta renda nas regiões centrais pela predominância de condomínios verticais. O baixo desempenho nas regiões periféricas (zonas norte e sul), por sua vez, pode ser explicado pela alta concentração da população de baixa renda (ARAUJO; BARROS; QUEIROZ, 2018).

Para aumentar a acurácia, o modelo foi corrigido com base na atribuição da autocorrelação à variável dependente (Spatial Lag), como sugerido pelos resultados dos testes de dependência espacial (Tabela 5). Os resultados são exibidos na Tabela 7. 
Tabela 7- Dados do Spatial Lag.

\begin{tabular}{|c|c|}
\hline Renda & $\mathbf{R}^{2}$ \\
\hline Sem renda & 0,014402 \\
\hline 1 a 2 SM & 0,332898 \\
\hline 2 a $5 \mathrm{SM}$ & 0,113437 \\
\hline 5 a $10 \mathrm{SM}$ & 0,610071 \\
\hline 10 a $20 \mathrm{SM}$ & 0,674372 \\
\hline acima $20 \mathrm{SM}$ & 0,683259 \\
\hline
\end{tabular}

Fonte: Os autores (2021).

Desta maneira, observando os valores de $\mathrm{R}^{2}$, podemos afirmar que o modelo corrigido foi capaz de prever $67 \%$ (10 a $20 \mathrm{SM}$ ) e 68\% (>20 SM) da localização da população de alta renda a partir da presença de piscinas.

Esses resultados foram considerados distintos dos obtidos por Vidal, Domene e Sauri (2011). Decorrência provável das diferenças dos contextos socioeconômicos, a densidade de piscinas de Marília mostrou maior associação com a renda do chefe do domicílio acima de 20 SM. Essa relação entre renda e piscina foi menos evidente na Região Metropolitana de Barcelona, atenuada pela grande variedade de características entre as piscinas, que representam significativas diferenças de custos de construção e manutenção.

Dentre os aspectos comuns de ambas as pesquisas, destaca-se a observação das desigualdades e das características do processo de urbanização. Os domicílios que possuem piscina, muitas vezes integrados aos jardins, podem ser considerados como privilegiados da perspectiva do tamanho do lote, característica comum do estilo de vida suburbano. E o desenvolvimento urbano segregado e de baixa densidade, característica dos subúrbios e condomínios fechados (CALDEIRA, 2000), pode ser considerado um importante atributo do processo recente de urbanização. Independentemente das diferenças entre o valor da moeda, renda média e dos estratos sociais das cidades brasileira e espanhola, acredita-se pertinente a afirmação de Vidal, Domene e Sauri (2011) de que a mencionada desigualdade da distribuição de bens simbolizem o prazer privatizado incorporado nas piscinas em meio aos problemas coletivos representados pelo aumento dos períodos de estiagem e dificuldades circunstanciais de abastecimento de água.

Explorar procedimentos alternativos para minimizar a defasagem de dados socioeconômicos nos períodos pré-censitários, pode ser considerada outra contribuição da pesquisa, pois permitiu inferir a localização da população de alta renda a partir de dados pontuais de Sensoriamento Remoto. Contudo, o uso destes procedimentos não deve ser considerado universal, em função da variabilidade das condições geográficas e climáticas das altas e baixas latitudes do globo terrestre. Deve ser complementado com outros métodos de análise, pois parte significativa das piscinas é interna nos climas subpolar e temperado.

Estes procedimentos podem ser aplicados em outras áreas de estudo e aprimorados com tecnologias de Data Mining e Machine Learning, que possibilitam aumentar a acurácia, reduzir a subjetividade do intérprete e minimizar os recursos e custos humanos e computacionais (HALL et al., 2009; SIVARAM; RAMAR, 2010; FRANCISCO; ALMEIDA, 2012; PETROPOULOS; PARTSINEVELOS; MITRAKA, 2013; MAXWELL et al. 2015; MAXWELL; WARNER; FANG, 2018). O uso de dados do sensor LiDAR (Light Detection and Ranging), também, pode contribuir ao fornecer elementos da verticalização urbana, minimizando no modelo a observada subestimação da população de alta renda dos condomínios verticais.

\section{CONSIDERAÇÕES FINAIS}

O artigo quantificou a relação entre a presença de piscinas e o nível socioeconômico da população de Marília. O objetivo era investigar o quanto a presença das piscinas poderia prever a localização da população de nível socioeconômico mais elevado. Foram usadas técnicas de GEOBIA, para classificar as piscinas, e de regressão linear e GWR para quantificar a relação entre as variáveis. 
Os resultados do GWR e Spatial Lag demonstraram que as duas variáveis: piscinas e renda estão estatística e espacialmente relacionadas. A estimativa de predição da localização do estrato de alta renda pela presença de piscinas foi de $67 \%$ para renda entre 10 e 20 SM e $68 \%$ para populações com renda de 20 SM ou mais.

Esta abordagem contribuiu para inferir a localização da população da alta renda a partir de dados pontuais de Sensoriamento Remoto. E, de certa forma, para reduzir a dependência dos dados socioeconômicos nos períodos que antecedem os censos demográficos decenais. Pode também ser aplicada em outras áreas de estudo e aprimorada em pesquisas futuras. Técnicas de automação como Data Mining e Machine Learning e dados de LiDAR podem ampliar a aderência e melhorar o desempenho do modelo.

\section{Agradecimentos}

Os autores agradecem ao Conselho Nacional de Desenvolvimento Científico e Tecnológico (CNPq) (Projeto Universal - Processo: 408077/2016-6) pelo financiamento da pesquisa.

\section{Contribuição dos Autores}

A autora ILS foi responsável pela redação da minuta inicial, visualização e curadoria dos dados. A autora ASA realizou a conceptualização, supervisão e redação da minuta inicial. $\mathrm{O}$ autor APQ contribuiu com a revisão e edição do texto, supervisão e aquisição de financiamento.

\section{Conflitos de Interesse}

Os autores declaram que não há conflitos de interesse.

\section{Referências}

ABOUD NETA, S. R.; SOUZA B. E.; DOS SANTOS, C. A. M. Classificação Baseada em Objetos e Mineração de Dados: Aplicação de Ferramentas Open Source no Mapeamento de Área Urbana. Revista Brasileira de Cartografia, Uberlândia- MG, v. 70, n. 3, p. 932-966, 2018. DOI. 10.14393/rbcv70n345707

ANSELIN, L. Spatial Econometrics. In: BALTAGI, B. H. A companion to theoretical econometrics. 2 ed. Boston: Kluwer Academic Publishers, 2001, p. 310- 330

Local indicators of spatial association-LISA. Geographical analysis, Columbus- EUA, v. 27, n. 2, p. 93-115, 1995. DOI. 10.1111/j.1538-4632.1995.tb00338.x

Lagrange Multiplier Test Diagnostics for Spatial Dependence and Spatial Heterogeneit. Geographical Analysis, Ohio- USA, v. 20, n. 1, p. 1-17, 1988. DOI. 10.1111/j.15384632.1988.tb00159.x

ARAUJO, A. S.; BARROS, J. X.; QUEIROZ, A. P. Segregação urbana: abordagem dos Índices sociais globais e locais. Revista do Departamento de Geografia, São Paulo- SP, v. spe, n. 1, p. 1-14, 2018. DOI. 10.11606/rdg.v0ispe.144092

QUEIROZ, A. P. Spatial Characterization and Mapping of Gated Communities. ISPRS International Journal of Geo-Information, v. 7, n. 7, p. 248- 260, 2018. DOI. 10.3390/ijgi7070248

AVELAR, S.; ZAH, R.; TAVARES, C. C. Linking socioeconomic classes and land cover data in Lima, Peru: Assessment through the application of remote sensing and GIS. International Journal of Applied Earth Observation and Geoinformation, Basel- Switzerland, v. 11, n. 1, p. 27-37, 2009. DOI. 10.1016/j.jag.2008.05.001

BAATZ, M.; SCHAPE, A. Multiresolution Segmentation: An Optimization Approach for High Quality MultiScale Image Segmentation. In: STROBL, J.; BLASCHKE, T.; GRIESBNER, G. Applied Geographische Informations-Verarbeitung. Ed. 3. Wichmann Verlag, Karlsruhe, Germany, p. 12-23, 2000.

BELGIU, M.; DRĂGUȚ, L.; STROBL, J. Quantitative evaluation of variations in rule-based classifications of 
land cover in urban neighbourhoods using WorldView-2 imagery. ISPRS Journal of Photogrammetry and Remote Sensing, v. 87, n. 1, p. 205-215, 2014. DOI. 10.1016/j.isprsjprs.2013.11.007

BURNETT, C.; BLASCHKE, T. A multi-scale segmentation/object relationship modeling methodology for landscapes analysis. Ecological Modelling, Oxfordshire, United Kingdom, v. 168, n. 3, p. 233-249, 2003. DOI. 10.1016/S030 4-3800(03)00139-X

BRUNSDON, C.; FOTHERINGHAM, A. S.; CHARLTON, M. E. Geographically weighted regression: a method for exploring spatial nonstationarity. Geographical analysis, Colombus- EUA, v. 28, n. 4, p. 281298, 1996. DOI. 10.1111/j.1538-4632.1996.tb00936.x

CALDEIRA, T.P. City of Walls: Crime, Segregation, and Citizenship in São Paulo. Ed. 1. University of California Press: London, UK, 2000.

CARVAlHO, L. E. X.; SILVA, H. N.; LOUREIRO, C. F. G.; MENESES, H. B. Regressão linear geograficamente ponderada em ambiente SIG. Revista Transportes, São Paulo, v. 14, n . 2, p.18-26, 2006. DOI. 10.14295/transportes.v14i2.65

COLARES, I. V. V.; NUNES, M. O.; SOUSA, G. M.; FERNANDES, M. C. Aplicação de GEOBIA para classificação da cobertura da terra no Parque Nacional do Itatiaia. Revista Brasileira de Geomática, v. 3, n. 1, p. 24-31, 2015.

CONGALTON, R. G. Assessing the accuracy of remotely sensed data: principles and practices. Ed. 3. Taylor \& Francis Group, Oxfordshire - United Kingdom, 2009.

COSTA, H.; FOODY, G.M.; BOYD, D.S. Supervised methods of image segmentation accuracy assessment in land cover mapping, Remote Sensing of Environment, v. 205, p. 338-351, 2018. DOI. 10.1016/j.rse.2017.11.024

CPTEC/INPE. Análise Climática de Marília/SP. Disponível em:< https://pt.climate-data.org>. Acesso em: 17 abr. 2020.

DOU, W.; REN, Y.; WU, Q.; RUAN, S.; CHEN, Y.; BLOYET, D.; CONSTANS, J. M. Fuzzy kappa for the agreement measure of fuzzy classifications. Neurocomputing, v. 70, n. 4, p. 726-734, 2007. DOI. 10.1016/j.neucom.2006.10.007

FEITOSA, F. F.; CAMARA, G. MONTEIRO, A. M. V., KOSCHITZKI, T.; SILVA, M. P. Global and local spatial indices of urban segregation. International Journal of Geographical Information Science, v. 21, n. 3, p. 299-323, 2007. DOI. 10.1080/13658810600911903

FERREIRA, M. Análise da sensibilidade dos testes de normalidade de Jarque-Bera e lilliefors em modelos de regressão linear. Revista Brasileira de Matemática e Estatística, São Paulo, v. 24, n. 4, p. 89-98, 2006.

FRANCISCO, C. N., ALMEIDA, C. M. Data Mining Techniques and Geobia Applied to Land Cover Mapping. Internacional Conference on Geographic Object-Based Image Analysis, 4, 2012. Proceedings of the 4th GEOBIA, Rio de Janeiro, p. 89-94, 2012.

FREITAS, M. W. D.; SANTOS, J. R. Esboço de modelagem da dinâmica da paisagem no Município de LagesSC. XV SIMPÓSIO BRASILEIRO DE SENSORIAMENTO REMOTO, 2011, Curitiba. INPE (Instituto Nacional de Pesquisas Espaciais, 2011 p. 67-42.

FOODY, G. M. Explaining the unsuitability of the kappa coefficient in the assessment and comparison of the accuracy of thematic maps obtained by image classification. Remote Sensing of Environment, v. 239, n. 1, p. 11- 11, 2020. DOI. 10.1016/j.rse.2019.111630

FOTHERINGHAM, A. S.; CHARLTON, M. E.; BRUNSDON, C. Spatial variations in school performance: a local analysis using geographically weighted regression. Geographical and Environmental Modelling, Oxfordshire- United Kingdom, v. 5, n. 1, p. 43-66, 2001. DOI. 10.1080/13615930120032617

GOUDARD, B.; OLIVEIRA, F. H.; GERENTE, J. Avaliação de modelos de localização para análise da distribuição espacial de unidades básicas de saúde. Revista Brasileira de Cartografia, Uberlândia- MG, v. 67, n. 1, p. 16- 34, 2015.

HAGEN-ZANKER, A. An improved Fuzzy Kappa statistic that accounts for spatial autocorrelation. International Journal of Geographical Information Science, v. 23, n. 1, p. 61-73, 2009. DOI. 


\section{$10.1080 / 13658810802570317$}

HALL, M.; FRANK, E.; HOLMES, G.; PFAHRINGER, B.; REUTEMANN, P.; WITTEN, I. H. WEKA Data Mining software: An update. ACM SIGKDD explorations newsletter- Association for Computing Machinery, New York- USA, v. 11, n. 1, p. 10-18, 2009. DOI. 10.1145/1656274.1656278

HAY, G. J.; CASTILLA, G. Geographic Object-Based Image Analysis (GEOBIA): A new name for a new discipline. In: BLASCHKE, T.; LANG, S.; Hay, G. J. Object-Based Image Analysis - Spatial Concepts for Knowledge-driven Remote Sensing Applications. Ed. 1. Berlim, Springer, 2006, p. 75 a 89. DOI.10.1007/978-3-540-77058-9_4

INSTITUTO BRASILEIRO DE GEOGRAFIA E ESTATÍSTICA (IBGE). In: Cidades, 2010. Disponível em: $<$ https://cidades.ibge.gov.br/>. Acesso em: 20 abr. 2020.

JARQUE, C. M.; BERA, A. K. Efficient tests for normality, homoscedasticity and serial independence of regression residuals. Economics Letters, Amsterdã, v. 6, n 3, p. 255-259, 1980. DOI. 10.1016/01651765(80)90024-5

JENSEN, J. R.; COWEN, D. C. Remote sensing of urban/suburban infrastructure and socioeconomic attributes. Photogrammetric Engineering and Remote Sensing, Oxfordshire-United Kingdom, v. 65, n. 1, p. 611-622, 1999.

KERR, G. H. G; FISCHER, C.; REULKE, R. Reliability assessment for remote sensing data: Beyond Cohen's kappa. IEEE INTERNATIONAL GEOSCIENCE AND REMOTE SENSING SYMPOSIUM (IGARSS). 2015, Milan- Italy, IEEE. p. 4995-4998, 2015. DOI. 10.1109/IGARSS.2015.7326954

KIM, M.; WARNER, T. A.; MADDEN, M.; ATKINSON, D. S. Multi-scale GEOBIA with very high spatial resolution digital aerial imagery: scale, texture and image objects. International Journal of Remote Sensing, v. 32, n. 10, p. 2825-2850, 2011a. DOI. 10.1080/01431161003745608

KIM, M.; HOLT, J.B.; EISEN, R.J.; PADGETT, K.; REISEN, W.K.; CROFT, J.B. Detection of swimming pools by geographic object-based image analysis to support west Nile virus control efforts. Photogramm. Eng. Remote Sensing, v. 77, p. 1169-1179. 2011b. DOI. 10.14358/PERS.77.11.1169

KRESSLER, F. P.; STEINNOCHER, K. Image data and LIDAR-an ideal combination matched by object oriented analysis. Geographic Object-Based Image Analysis, Salzburg, v. 36, n. 1, p. 36-60, 2006.

KONG, C., XU, K., WU, C. Classification and extraction of urban land-use information from high-resolution image based on object multi-features. Journal of China University of Geosciences, China, n.17, v.2, p. 151-157, 2006. DOI. 10.1016/S1002-0705(06)60021-6

KOTTEK, M. GRIESER, J. BECK, C. RUDOLF, B. RUBEL, F. World map of the Köppen-Geiger climate classification updated. Journal Meteorologische Zeitschrift, Berlim- Heidelberg, v. 15, n. 3, p. 259-263, 2006. DOI. 10.1127/0941-2948/2006/0130

LANDIS, J. R.; KOCH, G. G. An application of hierarchical kappa-type statistics in assessment of majority agreement among multiple observers. Biomectrics, v. 2, n. 33, p. 363-374, 1977. DOI. 10.2307/2529786

LLAUSÀS, A.; HOF, A.; WOLF, N.; SAURÍ, D.; SIEGMUND, A. Applicability of cadastral data to support the estimation of water use in private swimming pools. Environment and Planning B: Urban Analytics and City Science, v. 46, n. 6, p. 1165-1181, 2019. DOI. 10.1177/2399808318756370

LI, W.; GUO, Q. A new accuracy assessment method for one-class remote sensing classification. IEEE Transactions on Geoscience And Remote Sensing, v. 52, n. 8, p. 4621-4632, 2013. DOI. 10.1109/TGRS.2013.2283082

LIU, X.; HE, J.; YAO, Y.; ZHANG, J.; LIANG, H.; WANG, H.; HONG, Y. Classifying urban land use by integrating remote sensing and social media data. International Journal Geographical Information Science, Oxfordshire, United Kingdom, v. 31, n. 8 p. 1675-1696, 2017. DOI. 10.1080/13658816.201 7.1324976

LUCHIARI, A. Identificação da cobertura vegetal em áreas urbanas por meio de produtos de sensoriamento remoto e de um sistema de informação geográfica. Revista do departamento de Geografia, São Paulo, v. 14, n. 1, p. 47-58, 2001. DOI. 10.7154/RDG.2001.0014.0005 
MAXWELL, A. E.; STRANGER, M. P.; WARNER, T. A.; RAMEZAN, C. A.; MORGAN, A. N.; PAULEY, C. E. Large-Area, High Spatial Resolution Land Cover Mapping Using Random Forests, GEOBIA, and NAIP Orthophotography: Findings and Recommendations. Remote Sensing, v. 11, n. 12, p. 1409-1429, 2019. DOI. 10.3390/rs11121409

; WARNER, T. A.; FANG, F. Implementation of machine-learning classification in remote sensing: An applied review. Taylor \& Francis Group, v. 39, n. 9, p. 2784-2817, 2018. DOI. 10.1080/01431161.2018.1433343

, WARNER, T. A.; STRAGER, M. P.; CONLEY, J. F.; SHARP, A. L. Assessing machinelearning algorithms and image-and lidar-derived variables for GEOBIA classification of mining and mine reclamation. International Journal of Remote Sensing, v. 36, n. 4, p. 954-978, 2015. DOI. 10.1080/01431161.2018.1433343

MA, L.; LI, M.; MA, X.; CHENG, L.; DU, P.; LIU, Y. A review of supervised object-based land-cover image classification. ISPRS Journal of Photogrammetry and Remote Sensing, v.130, p. 277-293. 2017. DOI. 10.1016/j.isprsjprs.2017.06.001

MA, Z.; REDMOND, R. L. Tau coefficients for accuracy assessment of classification of remote sensing data. Photogrammetric Engineering and Remote Sensing, v. 61, n. 4, p. 435-439, 1995.

MCFEETERS S.K. Using the Normalized Difference Water Index (NDWI) within a Geographic Information System to Detect Swimming Pools for Mosquito Abatement: A Practical Approach. Remote Sensing. v. 5, n. 7, p. 3544-3561. 2013. DOI. 10.3390/rs5073544

MELAZZO, E. S. Padrões de desigualdades em cidades paulistas de porte médio. A agenda das políticas públicas em disputa. 2006, p. 229. Tese de doutorado apresentada ao programa de pós-graduação da Universidade Estadual Paulista (Faculdade de Ciências e Tecnologia), Presidente Prudente, 2006.

MO, D. K., LIN, H., LI, J., SUN, H., XIONG, Y. J. Design and implementation of a high spatial resolution remote sensing image intelligent interpretation system. Data Science Journal, Londres, v. 4, n. 6, p. 445452, 2007. DOI. 10.2481/dsj.6.S445

MONTEIRO, A. M. V., CÂMARA, G., CARVAlHO, M. S., DRUCK, S. Análise espacial de dados geográficos. Embrapa, Brasília, 2004.

NETTER, J., WASSERMAN, W. Applied linear statistical models. Ed 7. Illinois: Irwin, 1977.

NUNES, M. Produção do espaço urbano e exclusão social em Marília-SP. 2007, 173 p. Dissertação de mestrado apresentada ao Programa de Pós-Graduação em Geografia da UNESP de Presidente Prudente, Presidente Prudente- SP, 2007.

PETROPOULOS, G. P.; PARTSINEVELOS, P.; MITRAKA, Z. Change detection of surface mining activity and reclamation based on a machine learning approach of multi-temporal Landsat TM imagery. Geocarto International, v. 28, n. 4, p. 323-342, 2013. DOI. 10.1080/10106049.2012.706648

PONTIUS JR, R. G.; MILLONES, M. Death to Kappa: birth of quantity disagreement and allocation disagreement for accuracy assessment. International Journal of Remote Sensing, v. 32, n. 15, p. 44074429, 2011. DOI. 10.1080/01431161.2011.552923

SALIM, A., LUCHIARI, A. Utilização de geotecnologias para inferências populacionais no espaço urbano do município de Guarulhos-SP. XVI SIMPÓSIO BRASILEIRO DE SENSORIAMENTO REMOTO, Foz do Iguaçu- PR, v. 16, p. 794-801, 2013.

SEADE. Sistema SEADE de projeções populacionais. Disponível em: <http://produtos.seade.gov.br/pro dutos/projpop/>. Acesso em: 28 abr. 2020.

SIVARAM, N.; RAMAR, K. Applicability of clustering and classification algorithms for recruitment data mining. International Journal of Computer Applications, v. 4, n. 5, p. 23-28, 2010. DOI. 10.5120/8231165

SOUSA, A. M. O.; PEREIRA, J. M. O. C.; MOTA, B. W. C.; SANTOS, J. R. Análise da distribuição de fogos ativos detectados pelo sensor ATSR 2 e sua relação com variáveis demográficas, ambientais e infraestruturais: comparabilidade entre métodos de regressão OLS e GWR. Revista Brasileira de Cartografia, 
Uberlândia- MG, v. 3, n. 64, p. 704-721, 2012.

SOUZA, Í., ALVES, C. D., de ALMEIDA, C. M., PINHO, C. M. D. Caracterização socioeconômica do espaço residencial construído utilizando imagens de alta resolução espacial e análise orientada a objeto. Geografia, Londrina- PR, v. 16, n. 1, p. 119-142, 2007.

I. M. Análise da estrutura do espaço intra-urbano para estimativa populacional intercensitária utilizando dados orbitais de alta resolução espacial. 2003, 108 p. Dissertação de mestrado apresentada ao programa de pós-graduação do Instituto Nacional de Pesquisas Espaciais, INPE, São José dos CamposSP, 2003.

SPOSITO, M. E. B. Cidades médias: espaços em transição. Expressão Popular, São Paulo, 2007.

SPOSITO, M. E. B.; GÓES, E. Espaços fechados e cidades: Insegurança urbana e fragmentação socioespacial. São Paulo: Editora da UNESP, 2013.

STÜRCK, J.; SCHULP, C.J.E.; VERBURG, P.H. Spatio-temporal dynamics of regulating ecosystem services in Europe - the role of past and future land use change. Applyed Geography, Amsterdã, v. 63, n. 1, p. 121-135, 2015. DOI. 10.1016/j.apgeog.2015.06.009

VIDAL, M.; DOMENE, E.; SAURI, D. Changing geographies of water-related consumption: Residential swimming pools in suburban Barcelona. Area, United Kingdom, v. 43, n. 1, p. 67-75, 2011. DOI. 10.1111/j.1475-4762.2010.00961.x

WEISBERG, S. Applied Linear Regression. Ed. 3, Minnesota, John Wiley \& Sons, 2005.

XUAN, H.; LI, S. The Nonstationarity Tests of Geographically and Temporally Weighted Regression Model. Journal of Gansu Sciences, China, v. 2, n. 1, 2012.

ZANDONADI, J. Novas Centralidades e Novos Habitats: Caminhos para a Fragmentação Urbana em Marília (SP). 2008. 253 p. Dissertação (Mestrado) - Programa de pós-graduação em Geografia da UNESP campus Presidente Prudente, Presidente Prudente-SP, 2008.

ZHANG, B.; SONG, M.; ZHOU, W. Exploration on method of auto-classification for main ground objects of Three Gorges Reservoir area. Chinese Geographical Science, China, v.2, n. 15, p. 157- 172, 2005. DOI.10.1007/s11769-005-0009-7

ZHANG, C.; HARRISON, P.A.; PAN, X.; LI, H.; SARGENT, I.; ATKINSON, P.M. 2020. Scale sequence joint deep learning (ss-jdl) for land use and land cover classification. Remote Sensing of Environmental, Amsterdam, v. 237, n. 1, p. 1- 17, 2020. DOI. 10.1016/j.rse.2019.111593

\section{Biografia do autor principal}

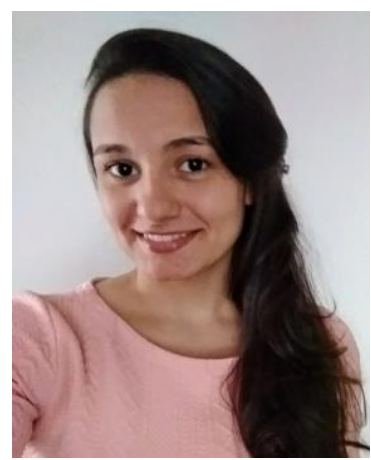

Mestranda em Geografia Física pela Universidade de São Paulo, possui Bacharelado e Licenciatura Plena em Geografia pela Universidade de São Paulo (2020). Tem experiência na área de Cartografia e Sensoriamento Remoto. Atua com Ensino de Geografia na Educação Básica. 